\section{Intersections}

Canadian Journal of Music

Revue canadienne de musique
Intersections CANADIAN JOURAL OR MUSIC

\title{
La genèse d'une reconnaissance réfléchie de la musique de film au Québec : le Prix Jutra
}

\section{Mathieu Lavoie}

Volume 30, numéro 2, 2010

URI : https://id.erudit.org/iderudit/1006377ar

DOI : https://doi.org/10.7202/1006377ar

Aller au sommaire du numéro

\section{Éditeur(s)}

Canadian University Music Society / Société de musique des universités canadiennes

\section{ISSN}

1911-0146 (imprimé)

1918-512X (numérique)

Découvrir la revue

Citer cet article

Lavoie, M. (2010). La genèse d'une reconnaissance réfléchie de la musique de film au Québec : le Prix Jutra. Intersections, 30(2), 41-60.

https://doi.org/10.7202/1006377ar
Résumé de l'article

Aux Jutra, concours récompensant l'industrie du cinéma québécois, on assiste aujourd'hui aux premières années d'une forme de reconnaissance réfléchie de la musique de film et des compositeurs oeuvrant dans ce domaine. C'est le résultat de nombreux débats - voire controverses — dans le milieu professionnel, en ce qui concerne la crédibilité du processus d'attribution des prix dans la catégorie " Meilleure musique ". En étudiant les résultats du concours depuis sa création en 1999 et en comparaison avec les règlements et l'historique des prix des Félix, des Génies et surtout des Oscars, nous montrerons que les mises à jour récentes des règles d'admissibilité des musiques aux Jutra sont justifiées, mais aussi qu'elles doivent être améliorées. L'un des objectifs de cet article sera de comprendre ce que représente la notion d'originalité en musique de film au regard d'une compétition dans l'industrie du cinéma québécois. Un deuxième objectif sera de questionner l'importance de reconnaître, dans des catégories distinctes, les musiques filmiques instrumentales « dramatiques » de celles davantage de style populaire ainsi que des chansons, lorsque celles-ci sont originales. Aussi, on s'interrogera sur l'impact de la popularité préétablie d'un artiste sur ses chances de remporter un prix pour son travail. Finalement, à partir des nouveautés aux Jutra de 2011, nous esquisserons certaines conséquences pour l'avenir de la compétition.
All Rights Reserved (C) Canadian University Music Society / Société de musique des universités canadiennes, 2011
Ce document est protégé par la loi sur le droit d'auteur. L’utilisation des services d’Érudit (y compris la reproduction) est assujettie à sa politique d'utilisation que vous pouvez consulter en ligne.

https://apropos.erudit.org/fr/usagers/politique-dutilisation/ 


\title{
LA GENĖSE D'UNE RECONNAISSANCE RÉFLÉCHIE DE LA MUSIQUE DE FILM AU QUÉBEC : LE PRIX JUTRA
}

\author{
Mathieu Lavoie
}

Aux Jutra, concours récompensant l'industrie du cinéma québécois, on assiste aujourd'hui aux premières années d'une forme de reconnaissance réfléchie de la musique de film et des compositeurs œuvrant dans ce domaine. C'est le résultat de nombreux débats - voire controverses - dans le milieu professionnel, notamment en ce qui concerne la crédibilité du processus d'attribution des prix dans la catégorie "Meilleure musique». Créé il y a treize ans à l'initiative des Rendez-vous du cinéma québécois «dans le but de récompenser les artisans et d'inciter le public à se rendre plus nombreux dans les salles ${ }^{1}$ », l'événement annuel, organisé par La grande nuit du cinéma ${ }^{2}$, ne disposait pas, jusqu'à tout récemment, de règlements définissant les critères d'admissibilité des musiques de film au concours. L'examen de certaines récompenses musicales attribuées depuis la création des prix Jutra témoigne d'une controverse entourant l'attribution d'un prix pour la musique préexistante et populaire de Dédé à travers les brumes en 2010, contre d'autres trames sonores constituées surtout de musiques originales, ainsi que d'importantes conséquences de celle-ci sur la crédibilité du concours. Mais l'une des conséquences, plus positive, est la mise en place de règles concernant l'admissibilité des bandes sonores à la compétition.

En effet, depuis le $13^{\mathrm{e}}$ gala des prix Jutra, en 2011, un minimum de $51 \%$ de la musique d'un film doit être original pour que celle-ci soit admissible à la compétition. Bien que la nouvelle règle ne soit pas encore publiée, elle constitue une première étape cruciale pour redorer la crédibilité du prix remis. De plus, la catégorie est passée de la «Meilleure musique» à la «Meilleure musique originale» afin de reconnaître avant tout le travail des compositeurs, puis celui des arrangeurs. Pour écrire ces règlements, il était nécessaire de comprendre ce que représente la musique de film pour les Québécois d'aujourd'hui, et plus particulièrement de s'interroger sur ce qui permet à une musique de film d'être considérée comme étant la «meilleure». L'un des critères avancés étant celui de l'originalité, notre objectif, dans cet article, sera de comprendre ce que représente cette notion dans le contexte de la musique de film. Pour ce faire, il faudra distinguer, dans des catégories distinctes, les musiques filmiques instrumentales «dramatiques» des musiques de style populaire, telles les chansons, lorsque

1 Lever et Pageau 2006, p.229.

2 Le concours est géré par l'organisme à but non lucratif La Grande Nuit du Cinéma et le gala est diffusé et coproduit par la Société Radio-Canada. 
celles-ci sont originales. En ce sens, l'examen des changements apportés aux règlements des catégories musicales des Academy Awards durant une période de 73 ans nous servira de référence. Nous comparerons ainsi les règlements et l'historique des prix des Félix, des Génies et surtout des Oscars - un examen qui montrera que si les mises à jour récentes des règles d'admissibilité des musiques aux prix de La grande nuit du cinéma se justifient, elles doivent néanmoins être améliorées. Cet examen nous incitera à nous interroger sur l'impact de la popularité préétablie d'un artiste sur ses chances de remporter un prix pour son travail, et à esquisser ainsi certaines conséquences pour l'avenir des Jutra.

Pour cet article, les concepts de musique «préexistante» et de musique «populaire» doivent être considérés d'une part d'une façon indépendante, et d'autre part, en relation l'un avec l'autre. Dans un film, une musique préexistante n'est pas systématiquement populaire ${ }^{3}$ comme une musique populaire n'est pas systématiquement préexistante 4 . Le terme "populaire» est une étiquette très accommodante, fixée non seulement sur les musiques ayant connu un réel succès commercial, mais aussi sur celles qui en auraient eu l'ambition. Ici, nous parlons de musique populaire d'une façon très inclusive. Le terme nous sert à représenter toute musique qui cherche essentiellement à plaire à une culture de masse dans sa période contemporaine et dans un territoire donné. Nous parlons de musique populaire par opposition à la «musique savante ${ }^{5}$ " et à la «musique folklorique ${ }^{6}$.

\section{UNE RECONNAISSANCE PROGRESSIVE EN MUSIQUE DE FILM}

Considérant que la musique de film existe depuis plus d'une centaine d'années au Québec, il n'est pas surprenant qu'un désir de la valoriser se manifeste depuis peu, notamment à la Société Professionnelle des Auteurs et des Compositeurs du Québec (SPACQ)7. En dehors des acclamations du public et de quelques articles de journaux, les musiciens œuvrant au cinéma québécois n'ont pas eu droit à une reconnaissance de leur travail avant plusieurs années. Le pianiste, compositeur et accompagnateur de cinéma muet Willy Eckstein, par exemple, décrit dans les publicités de son employeur, le Strand Theatre de

3 Les utilisations, par le réalisateur Denys Arcand, du $11^{\mathrm{e}}$ mouvement du Stabat Mater de Pergolessi, dans Jésus de Montréal (1989) et aussi, de l'air d'opéra "Che faro senza Euridice» d'Orfeo et Euridice, de Gluck, dans Réjeanne Padovani (1973) constituent des exemples de musiques préexistantes non populaires au cinéma.

4 Par exemple, la chanson populaire «Les Boys» a été composée par Éric Lapointe pour le film du même nom en 1998.

5 Voir: Tagg 1982, p.4. Selon Tagg, une musique savante, telle la musique classique, est principalement produite et transmise par des professionnels, peu souvent distribuée massivement et transmise par notation musicale. Contrairement à une musique folklorique, la musique n'est pas anonyme.

6 Idem. Selon Tagg, la musique folklorique serait de tradition écrite, rarement très répandue, produite généralement par des amateurs anonymes.

7 Nous ferons état, plus loin dans cet article, de l'implication récente de la SPACQ dans l'amélioration du processus de reconnaissance de la musique de film aux Jutra. 
Montréal, comme «le meilleur interprète de films de cinéma du monde ${ }^{8}$ » dont la musique, "bien souvent plus populaire que le film même ${ }^{9}$ ", aura accompagné le cinéma québécois pendant 24 ans ${ }^{10}$ sans jamais recevoir une quelconque reconnaissance officielle.

Maurice Blackburn, Eldon Rathburn, Robert Fleming et Louis Applebaum, qui formaient le moteur musical de l'Office national du film à Ottawa puis à Montréal à partir des années 1940, composaient des centaines de bandes sonores filmiques sans obtenir une mention au générique de façon régulière ${ }^{11}$. Ce n'est qu'en 1968 que les Canadian Film Awards, créés en 1949, remettent le premier trophée pour une musique filmique à l'Ontarien Louis Applebaum ${ }^{12}$. Trois ans plus tard, pour sa musique de Mon oncle Antoine, c'est Jean Cousineau qui devient le premier Québécois de naissance et le premier francophone ${ }^{13}$ à se faire reconnaitre pour sa musique de film lors d'une cérémonie pancanadienne. Les Jutra ont vu le jour exactement un demi-siècle après le début des Canadian Film Awards, mais ce n'est que récemment qu’un organisme québécois a offert une reconnaissance honorifique à un compositeur de musique de film. En 2004, le compositeur Richard Grégoire a reçu le prix Jutra hommage pour l'ensemble de sa contribution musicale au cinéma. Cette distinction devenait le point de départ de la reconnaissance publique du métier québécois de compositeur de musique de film. Aux États-Unis, l'équivalent s'était produit plus tôt, en 1985, alors qu'on remettait pour la première fois un Oscar honorifique à un compositeur, Alex North ${ }^{14}$. D’autres événements ont marqué la musique de film au Québec, mais c'est celui de 2004 qui retient toute notre attention car il s'agissait d'une consécration officielle du métier de compositeur de musique de film au Québec.

Cependant, l'hommage rendu au travail de Richard Grégoire ne vient pas combler l'ombre qui entoure la définition même du trophée Jutra récompensant la «Meilleure musique» de film; depuis sa fondation, La grande nuit du Cinéma offrait le prix sans jamais avoir défini de critères de sélection précis.

8 Bibliothèque et Archives Canada/Collection de la musique. Le gramophone virtuel. Willie Eckstein, pianiste et compositeur (1888-1963). http://www.collectionscanada.gc.ca/ gramophone/o28011-1008-f.html (consulté le $1^{\text {er juin 2011) }}$

9 Idem.

10 De 1906 à 1930, soit l'année où il quitte le Strand Theatre, conséquence de la popularité croissante du cinéma sonore.

11 Selon Eldon Rathburn, lors d'un entretien privé à son domicile, à Ottawa, le 30 novembre 2007, en compagnie de Christian Lewis.

12 Le trophée a été à Louis Applebaum pour sa musique du film Athabasca. Le titre de la catégorie est «music score (non feature)».

13 Eldon Rathburn, récompensé d'un Canadian Film Award (ETROG) en 1970 pour sa musique du documentaire Fields of Space, soit un an avant Cousineau, était québécois d'adoption, ayant dû déménager dans la région montréalaise pour suivre l'Office national du film, parti d'Ottawa en 1956. Rathburn est toutefois originaire du Nouveau-Brunswick et unilingue anglophone.

14 North détenait, et détient toujours, le record pour le plus grand nombre de nominations aux Oscars sans aucune victoire dans la catégorie "Meilleure musique», soit 14 (15, si l'on inclut sa nomination pour "Unchained Melody» dans la catégorie «Meilleure Chanson» en 1955. En valorisant la contribution exceptionnelle du compositeur au cinéma hollywoodien, l'attribution de la statuette honorifique devenait également une bonne solution pour contrer les faiblesses d'un système de reconnaissance imparfait. 
Il faudra attendre le résultat du $12^{\mathrm{e}}$ gala pour que la question soit soulevée. En effet, une chanson, une musique préexistante, un arrangement ou une orchestration méritent-ils d'être glorifiés par l'unique gala québécois portant sur le cinéma à l'affiche, aux dépens des musiques de film dramatiques écrites à l'image? Quels sont les critères d'admissibilité pour qu'une œuvre musicale filmique puisse passer à la postérité par le biais d'une reconnaissance au gala? La mise à jour des règlements et le gala de 2011 témoignent d'une profonde réflexion sur ce sujet.

\section{DÉDÉ À TRAVERS LES BRUMES}

Le gala des Jutra du 28 mars 2010 aura servi de catalyseur en invitant à une réflexion importante concernant la musique écrite pour le cinéma. Le prix pour la «Meilleure musique de film» a été attribué à Éloi Painchaud et aux Colocs pour la musique du film Dédé à travers les brumes, réalisé par Jean-Philippe Duval. On précise toutefois que ce prix est accordé "pour l'orchestration et la réalisation musicale ${ }^{15}$ ». Parmi les défaits, on trouve la musique originale semi-orchestrale du film Grande Ourse, la clef des possibles (Patrice Sauvé), composée par Normand Corbeil. Benoit Charest, compositeur des Triplettes de Belleville, se trouve aussi parmi les perdants, avec sa bande musicale épurée du drame Polytechnique, réalisé par Denis Villeneuve. En coulisse, la grogne discrète vécue par certains compositeurs québécois de musique de film aurait pu passer inaperçue auprès du grand public et de la presse. Selon le compositeur Michel Corriveau ${ }^{16}$, nommé deux fois pour un prix Jutra ${ }^{17}$, « un sentiment de frustration existe, surtout venant du fait qu'on a souvent eu l'impression que le prix [Jutra] avait plutôt les allures d'un concours de popularité, sans tenir compte de l'apport dramatique véritable de la musique au film, au montage et à l'histoire ${ }^{18}$ ». Pierre-Daniel Rheault, président sortant de la Société Canadienne des Auteurs, Compositeurs et Éditeurs de Musique (SOCAN) ${ }^{19}$ et professeur invité à la Faculté de musique de l'Université de Montréal, ajoute que «jusqu'en 2010, c'est certain qu'on grognait. [...] Avant [le gala de 2011], il y a eu des jeux de connaissance et de copinage qui ont fait que des compositeurs ont été disqualifiés sans justification ${ }^{20} »$. Richard Grégoire ajoute que plusieurs compositeurs, lui y compris, «ont trouvé étrange que le [Jutra de 2010] aille à Dédé Fortin ${ }^{21} »$.

15 Finalistes et gagnants de la $12^{\mathrm{e}}$ soirée des Jutra. 2010. http://www.lesjutra.ca/nominations.php (consulté le 14 septembre 2010)

16 Corriveau s'est notamment distingué par ses musiques des films, telles que celles pour Piché: entre ciel et terre (2010), Bon Cop, Bad Cop (2006) et Nez Rouge (2003). On lui doit aussi les musiques de nombreuses téléséries, dont Les Lavigueur (2008), ainsi que celles de plus de 500 publicités. Voir http://michelcorriveau.ca pour plus d'informations. Consulté le 30 mai 2011.

17 Pour ses musiques des films Le Survenant (2005) et Le Dernier Tunnel (2004)

18 Correspondance personnelle par courriel avec Michel Corriveau, le 30 mai 2011.

19 La SOCAN est l'organisme responsable de la gestion des droits d'exécution de la musique au Canada.

20 Entretien personnel avec Pierre-Daniel Rheault, par courriel, le $1^{\text {er }}$ juin 2011.

21 Correspondance personnelle par courriel avec Richard Grégoire, le $1^{\text {er }}$ juin 2011. 
La frustration du milieu n'a rien à voir avec la reconnaissance du travail de Painchaud et des Colocs, mais relève de l'originalité de la musique. En témoigne Richard Grégoire dans un entretien récent ${ }^{22}$. Michel Corriveau ajoute sa voix en écrivant :

Il n'y a aucun doute qu'une musique qui n’a pas été composée spécifiquement pour un film n'a pas lieu d'être récompensée. Un film qui ne contient pas de musique originale est amputé d'un artisan. Donc on ne peut récompenser quelqu'un qui n'a fait aucun geste artistique pour participer à la création de ce film ${ }^{23}$.

Est-ce que la sélection de musiques préexistantes doit être considérée comme un "geste artistique»? Si c'est le cas, devons-nous aussi récompenser, dans une tierce catégorie, les œuvres cinématographiques qui font un usage exceptionnel de musique préexistante, comme Dédé à travers les brumes? Certains films, notamment The Big Chill (1983) ${ }^{24}$, réalisé par Lawrence Kasdan, sont passés à la postérité pour leur utilisation de musiques préexistantes à fort pouvoir évocateur sans qu'un prix ne leur soit attribuée ${ }^{25}$. Le fait que certaines pièces préexistantes réussissent à évoquer, par association extracontextuelles ${ }^{26}$, des idées extramusicales ${ }^{27}$, peut expliquer pourquoi plusieurs cinéastes, dont Woody Allen et Stanley Kubrick, choisissent de n'utiliser que très rarement des musiques originales. De même Denys Arcand ira jusqu'à remettre en question le besoin de collaborer avec un compositeur lorsqu'une musique préexistante, qui répond à toutes ses exigences artistiques, est sur le marchée ${ }^{28}$.

\section{LES CONCOURS}

L'étude approfondie de l'évolution des règlements aux Oscars, aux Félix et aux Génies offre certaines solutions qui pourraient être appliquées pour améliorer la compétition des prix Jutra.

Pour ce qui est des Oscars, selon Fred Karlin dans son livre intitulé Listening to Movies $^{29}$, un débat sur la reconnaissance de la musique de film a déjà été ouvert. En effet, aux États-Unis, l'Academy of Motion Picture Arts and Sciences se penche sur la question des critères d'admissibilité des œuvres en

22 Correspondance personnelle par courriel avec Richard Grégoire, le $1^{\text {er }}$ juin 2011.

23 Correspondance personnelle par courriel avec Michel Corriveau, le 30 mai 2011.

24 Voir Lack 1997, p. 291-293.

25 Selon la base de données Internet Movie Database. www.imdb.com (consulté le 6 juin 2011).

26 Par association avec un élément extradiégétique, soit une époque, un lieu, un objet, un événement, etc.

27 Par exemple, un pays, un lieu, une époque, un objet, etc. La capacité d'une musique préexistante à évoquer des idées extramusicales est relative au niveau d'exposition des composantes musicales et sonores qui la constituent dans un ensemble démographique donné et à une époque précise. L'insertion de la chanson «La vie en rose» d'Édith Piaf dans un film, par exemple, insinue immédiatement un lieu, un personnage ou un événement lié à la France.

28 Lors d'un entretien public avec son biographe Réal La Rochelle intitulé «Conférence avec Denys Arcand et Réal La Rochelle». Faculté de musique de l’Université de Montréal. 9 novembre 2010 à la salle Serge-Garant. L'auteur était l'organisateur de l'activité, au nom de la SPACQ.

29 Karlin 1994, p. 207-220. Karlin fait un excellent survol historique des règlements relatifs à la musique aux Oscars. 
compétition depuis plusieurs années. Non seulement l'Academy a-t-elle reçu simultanément jusqu'à trois catégories pour la musique, faisant ainsi la distinction entre musique originale, chanson et comédie musicale filmique, mais fait-elle à présent l'effort de les définir publiquement. Ainsi, pour la course aux Oscars de l'année 2010, une partition filmique originale doit être constituée "d'une quantité substantielle de musique originale qui sert de trame dramatique soulignant le narratif, écrite spécifiquement pour le film par le compositeur qui la présente ${ }^{30}$ ». Les organisateurs prennent aussi soin de préciser que «les trames musicales diluées par l'utilisation de thèmes ou de musiques préexistantes, diminuées en impact par l'utilisation prédominante de chansons ou assemblées à partir de la musique de plus d'un compositeur, ne seront pas éligibles ${ }^{31}$ ». De plus, les Academy Awards indiquent que "l'éligibilité d'une œuvre sera évaluée selon son efficacité, sa finition, son degré de créativité et sa pertinence dramatique globale ${ }^{32} »$. Bien que, pour les Oscars, plusieurs efforts aient été déployés pour encourager les membres de l'Academy à voter d'une façon réfléchie, aucune règle ne sera jamais assez objective pour déterminer ce qui fait qu'une œuvre soit excellente. Néanmoins, les règles sont importantes car elles contribuent à la crédibilité du concours et aux résultats.

La distinction entre la musique originale et la musique préexistante existe depuis 1938 aux Oscars, soit quatre ans après la création du prix pour la musique. On passe ainsi de deux à trois catégories de récompenses, soit une pour Original score, une autre pour Music scoring, impliquant des arrangements de musiques préexistantes, provenant généralement de comédies musicales de Broadway, enfin une dernière pour Song, soit la meilleure chanson. Pour autant, l'histoire de la musique aux Oscars est parsemée d'incohérences, ainsi qu'en témoigne l'événement de 1938, par exemple, lorsqu'Alfred Newman remportait le prix pour la musique du film Alexander's Ragtime Band alors qu'il n'avait agi qu'en tant que chef d'orchestre33. Pour Karlin, les règles ont constamment été modifiées pour éviter qu'un tel événement ne se reproduise ${ }^{34}$. L'Academy s'est aussi souvent questionnée sur le degré d'originalité acceptable d'une bande sonore admissible à la compétition, ce qui s'observe notamment par l'ajout et le retrait des mots dramatic et original à la catégorie musicale dès 1941. Le mot original disparait au profit de la music score of a dramatic picture. Un an plus tard, on précise music score of a dramatic or comedy picture. Or en 1962, tout change : on élimine les précisions dramatic or comedy picture, concernant le type de film, pour revenir au concept de l'originalité en renommant la catégorie substantially

30 Academy of Motion Picture Arts and Sciences 2010, p. 18-19. Traduction libre de "An original score is a substantial body of music that serves as original dramatic underscoring and is written specifically for the motion picture by the submitting composer".

${ }^{1}$ Id., p.19. Traduction libre de "In addition, scores diluted by the use of tracked themes or other preexisting music, diminished in impact by the predominant use of songs, or assembled from the music of more than one composer shall not be eligible."

32 Academy of Motion Picture Arts and Sciences 2010, p. 19. Traduction libre de "The work's eligibility shall be evaluated on its effectiveness, craftsmanship, creative substance and relevance to the dramatic whole."

33 Karlin 1994, p.210-211.

34 Ibid., p. 210. 
original score. Mais quatre ans plus tard, l'Academy revient à sa dénomination d'original music score, soit la même que celle de 1938. Ensuite, en 1971, le mot dramatic réapparaît pour disparaître en 1975, revenir en 1995 et être supprimé en 1999. Depuis 13 ans, les Oscars proposent l'original score et l'original song. On ne récompense plus alors la meilleure musique pour une comédie musicale filmique dans une catégorie individuelle. Sont aussi exclues, depuis 1984, les adaptation scores (bandes musicales constituées d'arrangements musicaux et de musiques préexistantes), comme le seraient celles de Dédé à travers les brumes, C.R.A.Z.Y (2005) de Jean-Marc Vallée, Les amours imaginaires (2010) de Xavier Dolan et L'Enfant Prodige (2010) de Luc Dionne.

Pour ce qui est des Félix, l'Association québécoise de l'industrie du disque, $d u$ spectacle et de la vidéo (ADISQ) tient, depuis 1979, " un gala annuel visant à récompenser les artistes, artisans et professionnels de l'industrie québécoise de la musique ${ }^{35}$ ». Le gala offre une catégorie intitulée "Album de l'année bande sonore originale», depuis 1998. Contrairement aux Jutra, pour être admise au concours, la musique doit avoir « fait l'objet d'un produit distinct qui a été commercialisé comme tel», donc avoir été enregistrée sur disque, situation encore rare au Québec en matière de musique de film. Le Félix de la «Meilleure bande sonore originale» comporte une particularité importante et appréciable par rapport aux Jutra. Les règlements du concours spécifient qu'est admissible «toute trame musicale issue d'un film, d'une série télévisée ou autre production scénique ou littéraire dont $50 \%$ du contenu a été créé spécifiquement pour cette production ${ }^{36} »$. Ainsi, aux Félix, une bande sonore est considérée comme "originale» dès lors que la moitié de son contenu a été créée pour le film. Cette idée d'imposer une objectivité quantifiable à 50\% est-elle meilleure que la formule plus subjective des Oscars exigeant des membres du jury qu'ils évaluent les films en fonction de la quantité «substantielle» de musique? Un facteur additionnel, qui distingue les Félix des autres concours canadiens, tient compte du succès commercial de l'album : celui-ci vaut pour $40 \%$ du résultat, le reste étant déterminé par un vote parmi les membres de l'académie.

Une autre compétition permet aux compositeurs québécois d'obtenir une récompense pour leur travail filmique, soit le Gala des Prix Génie, organisé par l'Académie canadienne du cinéma et de la télévision (ACCT). L'organisme offre un trophée pour la «Meilleure musique originale» et, contrairement aux Jutra, un second prix récompensant la «Meilleure chanson originale». Les règlements publiés ${ }^{37}$ ne spécifient pas, comme c'est le cas des prix Félix, un pourcentage relatif à la proportion de contenu musical original minimum dans un film pour être éligible au concours. Toutefois, l'organisme prend soin d'exiger une feuille de mixage $3^{8}$ identifiant toutes les pièces musicales contenues dans le

35 Site internet de l'ADISQ. Http://www.adisq.com (consulté le 12 septembre 2010).

36 ADISQ. 2010. "Réglementation des Félix. Telle qu'adoptée par le conseil d'administration de l'ADISQ en date du 17 mars 2010 ".

37 ACCT. 2009. 31st Annual Genie Awards. 2010 Rules \& Regulations.

38 Feuille où l'on fait le dépouillement des musiques utilisées dans une production audiovisuelle. On y trouve généralement les informations relatives au film concernant le minutage des musiques, les compositeurs, les sociétés de gestion des droits d'auteurs, les éditeurs. Les feuilles de mixages précisent aussi les catégories d'utilisation des œuvres musicales contenues dans le film, telles que 
film ainsi que leurs durées respectives. Ce document aide les membres du jury à distinguer, lors du visionnement d'un film, les musiques préexistantes des musiques originales, ce qui diminue le risque de récompenser un compositeur pour une œuvre qui n'est pas la sienne.

\section{Originalité, CATÉgorie et POPUlarité}

Les règlements des prix Oscars, Félix et Génie sont tous publiés 39 et contiennent une clause plus ou moins détaillée concernant l'originalité des musiques admissibles aux concours. En 2010, ceux des Jutra ne se prévalaient d'aucune de ces caractéristiques, ce qui a permis à la musique du film Dédé à travers les brumes d'être récompensée. On ne remet pas en doute l'excellence de la musique des Colocs, ni le travail méticuleux et efficace d'Éloi Painchaud pour l'arrangement. Le problème est lié à l'absence de critères d'admissibilité : si le titre du prix était "Meilleure musique originale», comme modifié pour l'édition 2011 du concours, au lieu de "Meilleure musique ", la trame musicale de Dédé, constituée d'arrangement d'œuvres des Colocs déjà enregistrées sur disque, aurait été exclue de la compétition puisqu'elle n'est pas «nouvelle» et n’a pas été spécifiquement composée pour le film.

L'attribution d'un prix Jutra à la musique de Dédé à travers les brumes pose un nouveau problème, cette fois de nature sémantique. Ainsi, quelle interprétation doit-on donner à la notion de «musique» dans pareille circonstance? Une bande sonore filmique constituée de musique populaire, et plus précisément de chansons, peut-elle être jugée dans la même catégorie qu'une musique instrumentale composée pour les images? Selon le compositeur Michel Corriveau, «quelle que soit la forme de la musique proposée, si elle fait partie d'un processus créatif ayant pour but de faire partie du film [...] elle doit être considérée [admissible au concours $]^{40} »$. Le compositeur Luc St-Pierre, connu entre autre pour sa musique des téléséries Un tueur si proche (2004-aujourd'hui), Tribu.com (2001) ainsi que pour celle du film Prisoner of Paradise (2002), suggère «que la distinction devrait être faite, puisqu'il s'agit de champs de compétences proches, mais distincts ${ }^{41} »$. Le processus de composition d'une chanson ou d'une musique de style populaire est bien différent de celui d'une musique instrumentale dramatique, alors composée sur mesure, en synchronisme avec le film. La forme de l'œuvre peut faire figure d'exemple, prouvant la disparité entre les deux catégories musicales. Celle d'une chanson, par exemple, est généralement organisée autour du texte et rythmée par l'alternance couplets I refrains. Au contraire, la forme d'une musique instrumentale dramatique,

\footnotetext{
«musique de fond», «musique visuelle», «thème» et «logo». "Cue sheet», terminologie anglophone plus communément employée dans l'industrie du cinéma, est officiellement traduit par "feuille de mixage" par le Grand dictionnaire terminologique de l'Office québécois de la langue française, depuis 1976 (voir http://www.granddictionnaire.com). Toutefois, le formulaire de déclaration d'œuvres musicales utilisé en 2011 par la SOCAN est intitulé «Rapport de contenu musical/audiovisuel» et non pas «Feuille de mixage».

39 Sur les sites web des concours respectifs.

40 Correspondance personnelle par courriel avec Michel Corriveau, le 30 mai 2011.

41 Correspondance personnelle par courriel avec Luc St-Pierre, le 2 juin 2011
} 
composée pour un film, n'a rien d'aussi prévisible puisqu'elle s'indexe à celle du film et à sa narration. Cette différence pourrait expliquer pourquoi les Oscars distinguent les deux types d'œuvres en remettant deux prix différents.

La forte présence de la chanson dans le cinéma québécois indique que, tout comme aux Oscars, elle mérite une forme de reconnaissance individuelle au gala des Jutra. Au Québec, seulement cinq ans après la sortie du premier longmétrage de fiction, soit Le père Chopin (1945), «la chanson, celle qu'on entend sur le générique, celle qui identifie le film, fait son apparition dans Les lumières de ma ville, réalisé par Jean-Yves Bigras ${ }^{42} »$. Quatre chansons, composées par le chansonnier et réalisateur Pierre Pétel, s'y trouvent. Depuis, Gilles Vigneault, Robert Charlebois, Félix Leclerc, Stéphane Venne, Willie Lamothe, Michel Rivard et de nombreux autres artistes populaires québécois ont écrit pour le cinéma. Comme l'explique Robert Thérien, certaines de leurs chansons, tel Mon Pays de Gilles Vigneault composé pour le premier long métrage d'Arthur Lamothe en 1965, font désormais partie de notre patrimoine musical. Les compositeurs dits "généralistes 43 » d'aujourd'hui, œuvrant principalement dans le domaine de la musique à l'image, composent également des chansons populaires pour leurs bandes sonores. Michel Cusson a adapté son thème de Séraphin, un homme et son péché pour la chanson Depuis le premier jour, interprétée et popularisée par Isabelle Boulay. Mais selon Michel Corriveau, puisque «la profession [est] peu connue, on a tendance à attribuer à la vedette qui chante la chanson du générique le crédit de la musique du film ${ }^{44}$ ».

Lors de l'édition 2011 des Jutra, la distinction entre la chanson et la musique dramatique n'existe pas. Toutefois, le directeur général de la SPACQ, Jean-Christian Céré, fait actuellement des démarches pour qu'une catégorie intitulée "Meilleure chanson originale» y soit créée 45 . Cet ajout permettrait de sensibiliser le public au travail du compositeur dramatique qui écrit une musique en synchronie avec les images, par opposition à celui des auteurs et des compositeurs de chansons.

\section{VOTER POUR UNE MUSIQUE DE FILM}

Les démarches de la SPACQ concernant l'ajout de la catégorie «Meilleure chanson» et le changement apporté au titre de la catégorie "Meilleure musique» ne sont pas les premiers efforts effectués pour répondre aux détracteurs de la compétition des Jutra. Aux Jutra, un autre élément ayant fait l'objet de critiques et ayant nécessité une intervention de la part des organisateurs concerne le mode de votation. Selon Michel Corriveau, celui-ci serait responsable des

\footnotetext{
42 Robert Thérien. Livret du CD “Cinéma Cinéma — Les plus belles chansons du cinéma québécois". (Montréal : Musicor, 2008)

43 «Généraliste» signifie ici que le compositeur n’est pas nécessairement spécialisé dans un seul style - il est polyvalent. Il apprend les styles et les langages musicaux au fur et à mesure de ses commandes. Connu notamment pour sa musique de Bon Cop Bad Cop (2006), Michel Corriveau a écrit, au fil de sa carrière, des musiques rock, jazz, orchestrales, ethniques, technos ou des chansons.

44 Correspondance personnelle par courriel avec Michel Corriveau, le 30 mai 2011.

45 Conversation téléphonique avec Jean-Christian Céré, le 17 mai 2011.
} 
frustrations du milieu professionnel envers les Jutra ${ }^{46}$. Luc St-Pierre précise qu'il a «toujours trouvé ça curieux de recevoir annuellement le bulletin de vote d'une firme comptable [lui] permettant de voter sans même avoir vu un film ${ }^{47}$ ». Ainsi, pour améliorer la crédibilité de son scrutin, La grande nuit du cinéma mandate, depuis 2010, un jury pour visionner la totalité des films proposés et en sélectionner cinq qui seront mis en nomination. Par la suite, l'ensemble des membres de la SPACQ, association partenaire avec le gala, est invité à sélectionner, par courrier, le gagnant du trophée parmi les finalistes. Auparavant, ceux-ci étaient sollicités plus tôt dans le processus devant sélectionner quatre films pour la mise en nomination en remplissant un premier bulletin de vote. La grande quantité de films listés sur le bulletin de vote était problématique. Par exemple, vingt-sept films figuraient sur le Premier bulletin de vote pour le choix des finalistes ${ }^{48}$ de l'édition 2008 de La Soirée des Jutra. On peut lire sur ce bulletin que : «voter dans une catégorie sans avoir vu tous les films équivaudrait à une injustice envers les finalistes». En réalité, le visionnement de tous les films par un membre votant est un travail fastidieux, peut-être même impossible si l'on tient compte du temps considérable requis pour le réaliser, et de l'accessibilité parfois restreinte aux titres moins connus figurant parmi la sélection. L'injustice envers les finalistes, telle qu'écrite sur le bulletin de vote, résultant du non visionnement de l'ensemble des films par chacun des électeurs, est loin d'être unique à la compétition des Jutra et n'est pas récente. Concernant le système de votation par les pairs de l'Academy of Motion Picture Arts and Science, similaire à celui des Jutra, l'auteur Gene Ringgold écrivait, dans la revue Films in Review en 1961, qu'« en réalité, un grand nombre de membres vote sans avoir vu tous les films ${ }^{49}$ ».

\section{EXCELLENCE OU POPUlARITÉ?}

L'ajout d'un jury pour la sélection des finalistes depuis l'édition 2010 des Jutra fait état des efforts récents de l'organisme pour établir une forme de reconnaissance réfléchie concernant la musique de film et les compositeurs œuvrant dans ce domaine au Québec. Une réflexion plus approfondie est encore nécessaire pour définir si le prix Jutra doit être accordé pour la valeur artistique d'une œuvre musicale ou pour sa popularité.

Comme le précise le directeur général de l'organisation Henry Welsh, les Jutra «ne sont pas un concours d'excellence, mais plutôt l'expression des préférences de chacun ${ }^{50}$ ». Mais ne devraient-ils pas justement être un concours d'excellence en raison de leur influence sur la mémoire collective des québécois? Une critique musicale du Los Angeles Times, Isabel Morse Jones, faisait un constat similaire par rapport aux Oscars, en 1944: «un jour, les juges de l'académie auront l'instruction de choisir un film pour sa musique sans tenir

46 Correspondance personnelle par courriel avec Michel Corriveau, le 30 mai 2011.

47 Correspondance personnelle par courriel avec Luc St-Pierre, le 2 juin 2011

48 La Soirée des Jutra. Prix Jutra 2008 - Longs Métrages / Fictions. Premier Bulletin de vote pour le choix des finalistes. Catégorie $\mathrm{n}^{\mathrm{0}} 13$ «Meilleure Musique».

49 Cité dans : Karlin 1994, p. 215. Traduction libre.

50 Correspondance personnelle par courriel avec Henry Welsh, le 9 mai 2008. 
compte de sa popularité générale ${ }^{51}$ ». Les remises de prix contribuent à déterminer les œuvres qui passeront à la postérité ainsi qu'en témoigne le résultat d'un sondage effectué par l'American Film Institute dans le cadre de sa célébration du centenaire du cinéma en 2005. L'AFI a questionné plus de 500 artistes, compositeurs, musiciens, critiques et historiens pour sélectionner la liste des 25 meilleures musiques de film de tous les temps ${ }^{52}$. Mettant de côté King Kong, sorti en 1933, soit un an avant la création de l'Oscar pour la musique, ainsi que The Godfather, exclu in extremis du concours de 1973 lorsque l'on s'aperçut que sa mélodie principale avait déjà été utilisée par Nino Rota dans le film franco-italien Fortunella $(1958)^{53}$, 21 des 23 films restants ont reçu ou été nommés pour un Oscar dans la catégorie musicale. Les deux films exclus, Vertigo (1958) et Psycho (1960), ont en commun d'être réalisés par Alfred Hitchock et mis en musique par Bernard Herrmann. Comment comprendre cette exclusion? Labsence de la musique de Psycho à la compétition s'expliquerait par le fait qu'elle constitue un "départ notable des conventions en musique de film ${ }^{54}$ ». La proportion impressionnante de musiques de film figurant à la fois sur la liste des Oscars et sur celle de l'AFI indique que les résultats des cérémonies de remise de prix ont un impact historique important. La grande nuit du cinéma est, en effet, la seule institution en son genre au Québec, mais il n'en demeure pas moins qu'elle a une responsabilité morale et éthique et se doit de récompenser l'excellence avant la popularité et de justifier son processus d'attribution des prix au grand public auprès de son conseil d'administration, formé de représentants de diverses associations en lien avec l'industrie du cinéma, dont la SPACQ pour la musique.

Mais pour récompenser l'excellence, il faut tout d'abord savoir ce que l'on désigne. En quoi une musique est-elle «meilleure»? Le musicologue et compositeur Michel Chion rappelle que «la musique de quatre sous peut être, et pas seulement paraître, très belle au cinéma, pour des raisons cinématographiques 55 ». Refuser une musique de film parce qu'elle serait «simpliste» reviendrait à éliminer un très grand nombre des partitions filmiques. Selon PierreDaniel Rheault, évaluer l'excellence d'une ouvre est difficile, «cela réfère à la grande question sur l'esthétique. Une musique qui me plaît ne fera pas nécessairement l'unanimité. C’est tellement subjectif. [Pour mériter un prix], il faut que la musique soit techniquement irréprochable, tant au niveau de l'orchestration que dans la conduite des voix ${ }^{56} »$. Devrions-nous suivre l'exemple des Américains qui, rappelons-le, précisent, dans les règlements officiels des Academy Awards, que la "finition et le degré de créativité des œuvres seront considérés pour leur éligibilité au concours »? Ces questions doivent être débattues individuellement pour chaque concours, et les solutions proposées, même si

51 Isabel Morse Jones. Los Angeles Times (1944). Cité dans : Karlin 1994, p.209. Traduction libre de "Some day the [Academy judges will be instructed to choose picture for their music and pay no attention to their general popularity."

52 American Film Institute 2005. http://www.afi.com/10oyears/scores.aspx

53 AMPAS. Awards Database. http://awardsdatabase.oscars.org

54 Prendergast 1992, p.133. Traduction libre.

55 Chion 1995, p.18.

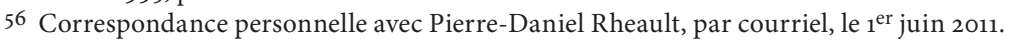


elles laissent une grande marge de manœuvre au jury, devraient invariablement être publiées dans leurs règlements officiels.

\section{LES JUTRA SOUS LA LOUPE : 1999-2003}

Les insatisfactions constatées entourant la reconnaissance en musique de film au Québec ne sont pas nées lors de la compétition des Jutra de 2010. La toute première musique de film ayant été récompensée d'un prix Jutra est celle de John Corigliano, pour le film Le Violon Rouge de François Girard. Le film était partiellement québécois ${ }^{57}$, mais la musique était américaine ${ }^{58}$. Se questionnant sur la pertinence de récompenser un compositeur étranger, Richard Grégoire précise que "Les Jutra sont là pour reconnaître les créateurs québécois». Mais Grégoire ajoute qu’au Québec, "on était bien fier que Benoit Charest soit sur la liste des compositeurs nommés aux Oscars, en 2004, avec les Triplettes de Belleville 59 ». Ainsi, si l'on considère que les Québécois sont admissibles à la compétition des Oscars, on ne devrait pas pouvoir exclure les compositeurs étrangers de la compétition des Jutra. Il est intéressant de noter que, lors de cette soirée, étaient aussi nommés Pierre Desrochers et Nathalie Boileau pour la musique du film Un 32 août sur Terre de Denis Villeneuve, principalement constituée de la musique de Robert Charlebois et de Jean Leloup ${ }^{60}$. Cette musique préexistante fait partie du patrimoine québécois, et cette popularité pourrait avoir eu un impact sur la mise en nomination de la bande sonore, notamment en raison de l'absence d'instructions pour les membres-votants.

En 2000, le compositeur Benoît Jutras reçoit le prix pour la musique du film Alegria, de Franco Dragone. Toutefois, le film comprenait un très grand nombre de pièces préexistantes, composées quelques années plus tôt par René Dupéré pour le spectacle du Cirque du Soleil, Alegria. Les électeurs, à moins d'être familiers avec l'œuvre de Dupéré, pouvaient difficilement dissocier sa musique préexistante de la musique nouvelle. Sans remettre en cause l'excellence de l'œuvre de Jutras, le film Alegria a-t-il remporté le Prix grâce à la musique de Jutras, grâce à celle de Dupéré ou aux deux? Le résultat de 2002 soulève un questionnement tout aussi important. Un crabe dans la tête, film de 102 minutes réalisé par André Turpin, ne compte, en effet, qu'un peu plus de sept minutes de musique, incluant celle accompagnant le générique final, et dont moins de cinq sont originales ${ }^{61}$. La pièce principale du film, entendue à trois reprises, n'est pas originale mais reprend une chanson traditionnelle italienne intitulée Bella Ciao. Considérant qu’aux Oscars, un film doit contenir «une quantité substantielle de musique originale» pour être admis à la catégorie «Meilleure musique», il est légitime de se demander, dans le cas des Jutra, en quoi une musique originale est-elle assez présente dans un film pour être

57 C'était une coproduction entre le Canada, l'Italie et le Royaume-Uni.

${ }^{8}$ John Corigliano est un américain ayant vécu à New York toute sa vie, selon son site internet personnel. http://www.johncorigliano.com (consulté le 13 septembre 2010)

59 Correspondance personnelle avec Richard Grégoire par courriel, le $1^{\mathrm{er}}$ juin 2011.

60 Voir Latreille 2008, p.91-108.

${ }^{61}$ Le film comprend une scène où le protagoniste remet, à une femme sourde, un carton sur lequel il est inscrit «Le silence me fascine», ce qui justifie peut-être le peu de musique. 
récompensée par un trophée? Un film de deux heures comportant seulement trois interventions d'un thème de 20 secondes, interprété par un violon seul, devrait-il être jugé à titre égal avec un film comportant 45 minutes de musique orchestrale? Comment déterminer la quantité minimale de musique originale dans un film pour que celle-ci soit acceptée au concours ${ }^{62}$ ? Pierre-Daniel Rheault avance qu' «une musique est jugée substantielle à partir du moment où elle contribue d'une façon considérable au déroulement dramatique, nonobstant sa durée $e^{63}$ ». Il serait difficile d'obtenir un consensus chiffré, mais une solution similaire à celle de l'Academy of Motion Picture Arts and Sciences, qui a choisi de ne pas donner de directive quantifiée à ses membres tout en leur rappelant qu'une quantité substantielle de musique est nécessaire, serait envisageable pour les Jutra.

\section{LES JUTRA DE 2004 À 2010 : UNE CONSTANTE}

Une constante s'observe en ce qui concerne les récipiendaires des prix entre 2004 et 2010. Durant ces sept années, les Jutra récompensant la «Meilleure musique» ont toujours été remis à des artistes œuvrant principalement dans un domaine autre que celui de la musique de film (sauf exception en 2009, décrite plus loin). De ce fait, pour qu'une bande sonore filmique soit reconnue comme étant la meilleure, on peut se demander si l'artiste qui l'a créée doit détenir une certaine popularité. Le système de votation par les pairs pénaliset-il les compositeurs moins connus? Bien entendu, la qualité et le mérite des bandes sonores couronnées, généralement excellentes, de même que les styles musicaux utilisés, ne sont pas ici remis en cause. Ce qui nous intéresse est de constater le phénomène afin de mieux le comprendre.

En 2004, l'harmoniciste Guy Bélanger et le guitariste Claude Fradette remportent le trophée pour leur musique de Gaz Bar Blues (Louis Bélanger) contre notamment Michel Cusson et Jean-Marie Benoît, deux compositeurs expérimentés. Un an plus tard, le chanteur Dumas et son proche collaborateur, le compositeur-interprète Carl Bastien, tous deux très connus dans le milieu de la musique populaire ${ }^{64}$, remportent un Jutra en faisant leur première incursion conjointe au cinéma pour le film Les Aimants d'Yves Pelletier. Dans la course au prix se trouvaient également trois ténors du métier de compositeur de musique filmique, Michel Corriveau (Le dernier tunnel), Michel Cusson (Dans une galaxie près de chez vous) et James Gelfand (Jack Paradise). Un autre chanteur populaire faisant sa première expérience au cinéma en tant que compositeur

62 La compétition de 2003 a récompensé Michel Cusson pour sa musique de Séraphin, un homme et son péché (Charles Binamé). En 13 ans de Jutra, seulement deux prix ont été accordés à des musiques faisant un fort usage d'un style orchestral, soit celui de Cusson et celui accordé à John Corigliano en 1999.

63 Correspondance personnelle avec Pierre-Daniel Rheault, par courriel, le $1^{\text {er }}$ juin 2011.

64 Dumas était récemment nommé dans les catégories Album de l'année - Rock et Interprète masculin de l'année au Gala de l'ADISQ de 2004. Carl Bastien, aussi nommé deux fois au Gala de l'ADISQ, était entre autres déjà connu pour ses collaborations avec Daniel Bélanger, Ariane Moffat et Marc Déry. 
principal ${ }^{65}$, Daniel Bélanger, remporte la compétition en 2006 pour sa musique du film l'Audition ${ }^{66}$ (Luc Picard). Le "poète intègre doublé d'un musicien ingénieux et innovateur ${ }^{67} »$, déjà très reconnu pour ses albums ${ }^{68}$, l'emporte sur la musique orchestrale dramatique de Michel Cusson, fort présente dans le film Maurice Richard (Charles Binamé). Jorane, déjà nommée 14 fois au gala de l'ADISQ et hautement reconnue pour l'originalité de ses chansons éclectiques alliant violoncelle et "voix liquide ${ }^{69}$ ", récolte le prix en 2007 , tout en demeurant dans son univers musical personnel, avec sa touchante musique du film Un dimanche à Kigali (Robert Favreau). Notons que pour cette compétition, elle assurait sa propre concurrence en étant également nommée pour sa bande sonore de Kamataki (Claude Gagnon). Tout comme Jorane, la récipiendaire du trophée de 2008 est elle aussi la principale interprète de sa trame sonore filmique et s'est fait connaître par sa musique populaire ${ }^{70}$. Pour le film Le Ring, réalisé par Anaïs Barbeau-Lavalette, les délicates interventions d'un thème valsé et de ses variations, interprétées par la compositrice au piano et accompagnées sobrement par quelques touches de contrebasse, trombone et guitare, font remporter le trophée à la jeune Catherine Major, de la scène émergente. Incluant son générique, le film compte un peu plus de 18 minutes de musique, réparties en une douzaine de tops, ce qui représente approximativement $20 \%$ de sa durée totale. C'est peu à la lumière de la cinquantaine de minutes de musique originale, réparties en une quarantaine de tops, du film Ma Fille, Mon ange (Alex Durand-Braut), en lice pour un Jutra la même année. Cette musique de Normand Corbeil correspond à $60 \%$ de la durée totale du film. Les 18 minutes de musique de Le Ring semblent insignifiantes si on les compare avec les productions hollywoodiennes. Mais il est difficile d'imaginer Le Ring avec davantage de musique; le caractère épuré de cette bande sonore s'harmonise délicatement avec l'esthétique introvertie du film. Il n'en demeure pas moins que la durée de la musique de Le Ring soulève un questionnement similaire à celui posé plus tôt pour Un crabe dans la tête : est-ce que son minutage musical doit être considéré comme «une quantité substantielle de musique originale»? La quantité est ici certainement très proportionnelle aux besoins du film, lui-même loin du stéréotype filmique hollywoodien.

65 Bélanger avait été nommé dans la catégorie «Meilleure chanson originale» à la compétition des prix Génie en 2000 pour la chanson thème du film Le Dernier Souffle.

66 Sa musique fut aussi mise en nomination pour la compétition des prix Génie de 2005 dans la catégorie «Meilleure chanson originale».

67 Annie Joan Gagnon. Daniel Bélanger. L’encyclopédie de la musique au Canada. http://www. thecanadianencyclopedia.com. Consulté le 31 mai 2011.

68 Dès 1993, l'année de la sortie de Les Insomniaques s'amusent, son premier album solo, Bélanger remporte le prix Félix pour le meilleur album pop-rock, en plus d'être nommé dans les catégories Auteur ou compositeur de l'année, Interprète masculin de l'année et deux fois dans la catégorie Vidéoclip de l'année. Son deuxième album, Quatre saisons dans le désordre (1996), est certifié platine. En 2002, son album Rêver mieux lui remporte sept Félix au gala de l'ADISQ et le prix Juno de l'album francophone de l'Année.

69 Alain De Repentigny. La Presse. 19 février 2010. Voir aussi : Marie-Christine Blais, La Presse, 18 Février 2010.

70 Rose sang, son deuxième album et premier succès commercial avec plus de 20 ooo exemplaires vendus, sort en en janvier 2008, soit deux mois avant la Soirée des prix Jutra qui la couronne pour sa musique du film Le Ring. http://catherinemajor.com (consulté le 31 mai 2011) 
Michel Corriveau écrit que "quelques minutes de musiques dans une trame dramatique peuvent suffire à insuffler un sous-texte extrêmement pertinent et dramatique [...] [alors que] certains films peuvent être remplis entièrement de musique sans aucune intention dramatique ${ }^{71}$ ». Mais si Le Ring n'avait eu que cinq minutes de musique originale, comme c'est le cas pour Un crabe dans la tête, aurait-ce été suffisant? Une bande sonore plus longue ne devrait-elle pas, en principe, représenter un effort plus considérable de la part du compositeur?

Quant à la compétition de 2009, elle récompensait le duo constitué d'un vétéran de la musique de film, Normand Corbeil, et d'un artiste populaire, Serge Fiori, l'ex-chanteur-guitariste du groupe notoire Harmonium, pour leur musique du film Babine réalisé par Luc Picard. À l'écoute, on reconnait le travail de Corbeil par les tenues très atmosphériques aux synthétiseurs, rappelant la musique de Grande Ourse, et celui de Fiori, par la forte présence de sa voix chantée, sans paroles et d'une guitare à 12 cordes.

\section{LES JUTRA DE 2011 : LES PREMIERS GRANDS PAS}

Ce tour d'horizon a tout d'abord permis de constater que la compétition des Jutra accusait, en 2010, un retard considérable par rapport à sa réflexion sur la musique de film et à sa responsabilité éthique sur le plan de la qualité de son dispositif d'évaluation. Nous avons établi que, en raison de ses résultats, le gala des prix Jutra influence la culture musicofilmique des Québécois, ce qui rend d'autant plus importante la crédibilité de son processus de mise en nomination. Cependant, en vue de l'édition 2011 de La soirée des Jutra, la SPACQ a tenté de remédier à la situation en créant un comité constitué de membres de compositeurs de musique à l'image, chargé de proposer de nouveaux critères d'admissibilité au concours La Grande Nuit du Cinéma. Benoît Charest et Guy Bélanger ont remporté le $13^{\mathrm{e}}$ concours dans la catégorie musicale pour la vingtaine de minutes de musique qu'ils ont composée pour Route 132 (Louis Bélanger). Ils deviennent ainsi les premiers à mériter le trophée avec sa nouvelle dénomination de "Meilleure musique originale». Un film absent de la liste de nominations retient cependant toute notre attention. Il s'agit du drame biographique L'Enfant Prodige, réalisé par Luc Dionne. Constituée de plus de $75 \%$ de musique préexistante d'André Mathieu, de Mahler, de Mozart et de Rachmaninoff, la bande sonore, contenant aussi la musique originale du pianiste Alain Lefebvre, ne cadre pas avec les nouveaux critères d'admissibilité de la compétition, et à l'heure actuelle, il est impossible de savoir si les membres du jury l'auraient placée dans la liste des finalistes en l'absence des nouveaux règlements. Sans la nouvelle règle, les Jutra de 2011 auraient pu répéter l'expérience de 2010, accordant le prix pour une bande sonore principalement constituée de contenu préexistant.

Le nouveau règlement exigeant $51 \%$ de musique originale aux prix Jutra doit être affiné. Selon Richard Grégoire, cette proportion semble raisonnable. Mais le compositeur précise "qu'il est important que le jury puisse bien comprendre, lors du visionnement, quelle musique est originale et laquelle vient d'une autre

71 Correspondance personnelle par courriel avec Michel Corriveau, le 30 mai 2011. 
source». En 2011, les producteurs n'avaient pas encore l'obligation de remettre une feuille de mixage de leur film aux organisateurs des Jutra, situation qui devrait être corrigée en 2012 selon Jean-Christian Céré, directeur général de la SPACQ et membre du conseil d'administration de La grande nuit du cinéma ${ }^{72}$. L'information se trouvant sur une feuille de mixage traditionnelle, telle celle envoyée par les producteurs de films à la SOCAN pour la gestion des droits d'exécution ${ }^{73}$, ne permet cependant pas de déterminer si une musique attribuée au compositeur du film a été composée spécifiquement pour le film ou si elle faisait déjà partie de son catalogue préexistant ${ }^{74}$.

On constate avec étonnement qu'une bande musicale de dix minutes constituée de $51 \%$ (donc cinq minutes) de musique originale serait admissible alors qu'une autre de 60 minutes constituée de $50 \%$ (30 minutes) de musique originale ne le serait pas. Ainsi, une bonne et substantielle musique originale pourrait se voir éliminer en raison d'une trop forte présence de musique préexistante. Cette règle a pour but d'éviter aux membres de la SPACQ d'être influencés par la musique préexistante lors du suffrage. Une solution serait qu'à partir d'une certaine durée raisonnable de musique originale, la règle soit contournée, peu importe la quantité de musique préexistante partageant l'espace sonore du film. Il faudra aussi décider si un minimum de musique originale, en durée, et non pas seulement proportionnellement à la musique préexistante, doit être fixé. La tâche n'est pas évidente, selon Michel Corriveau, puisqu'il «est tellement difficile d'établir des règles précises et de quantifier quelque chose qui relève des $\operatorname{arts}^{75} »$.

\section{L'AVENIR DES JUTRA}

Du travail reste encore à faire au Québec sur le plan de la compréhension, de la reconnaissance et de la mise en valeur des musiques de film locales, mais le processus est à présent enclenché. La progression exceptionnelle de la part des marchés des films québécois depuis une vingtaine d'années ${ }^{76}$ justi- $^{-}$ fie la nécessité d'établir un processus de sélection de plus en plus ciblé pour

72 Il est important de noter, toutefois, que bien qu'ils n'en aient pas l'obligation, les producteurs ont offert les feuilles de mixage de leurs films sur une base volontaire, à la demande de La grande nuit du cinéma. Il ne resterait qu'à officialiser le processus.

73 Soit le «Rapport de contenu musical», tel que décrit précédemment dans cet article.

74 Par exemple, la bande sonore de Yann Tiersen du film «Le fabuleux Destin d'Amélie Poulain » (2001) est d'une part constituée de musique provenant de son catalogue musical préexistant et, d'autre part, de musique composée exclusivement pour le film. Utilisant le «rapport de contenu musical» de la SOCAN, il serait impossible de faire la distinction entre la nouvelle musique de Tiersen et celle déjà enregistrée sur disque avant la production du film. Toutefois, le rapport de contenu musical aide les membres du jury à identifier les œuvres qui sont composées par d'autres compositeurs que ceux figurant au générique. Dans le cas d'Amélie Poulain, par exemple, le «rapport de contenu musical» permettrait facilement d'identifier que la pièce «The Child» est d'Alex Gopher et non pas de Tiersen, tout comme la pièce "Adagio for Strings" qui s'y trouve aussi est de Samuel Barber.

75 Correspondance personnelle par courriel avec Michel Corriveau, le 30 mai 2011.

76 Voir Trépanier-Jobin 2008. À la page 3, l'auteur affirme qu' "entre 1992 et 2006, la part de marché des films québécois a plus que quadruplé en suivant une croissance exponentielle...». On peut aussi lire que «les recettes en dollars actualisés et l'assistance des films québécois sortis en salle entre 2004 et 2006 sont sept fois plus importantes qu'elles ne l'étaient entre 1992 et 1994 ". 
déterminer les musiques filmiques qui vivront dans la mémoire collective de la société. Les galas peuvent être d'excellents moyens pour y parvenir, mais ceuxci doivent également poursuivre leur effort pour mieux définir les catégories et les critères d'admissibilité. Si l'on se fie à l'évolution des catégories musicales aux Oscars, la compétition des Jutra devrait poursuivre son questionnement pendant encore plusieurs années. Ces affinements auront l'avantage de limiter la confusion et les frustrations, avant et après les cérémonies. Mais plus les règles et les catégories deviendront précises, plus elles deviendront exclusives, mettant de côté certaines œuvres musicales exceptionnelles non admissibles à la compétition. Pour compenser, peut-être faudra-t-il un jour considérer la création d'un Gala des exclus? Un peu à l'image du Salon des Refusés? S'y trouveront, entre autres, les musiques québécoises de documentaires offerts uniquement sur le marché des DVD77. Les musiques composées pour les films publicitaires $^{78}$, actuellement dépourvues de système de reconnaissance local, les réalisateurs qui font une utilisation exceptionnelle de musique préexistante ainsi que les bandes sonores ne contenant qu'un faible pourcentage de musique originale pourraient aussi se voir décerner un trophée. Et pourquoi ne pas y inclure les musiques de jeux vidéo produites localement, de plus en plus présentes en réponse à la forte croissance de cette industrie 79 ? L'amélioration de la reconnaissance du talent des compositeurs québécois de musique de film devra se faire non seulement au travers de nouvelles mises à jour des règlements des concours et galas, mais aussi en valorisant davantage leur travail aux yeux du public. Pour ce faire, les systèmes de reconnaissance devront s'adapter, sur une base annuelle, selon l'évolution croissante de la profession et du marché.

\section{RÉFÉRENCES}

Academy of Motion Picture Arts and Sciences. 2010. "83rd annual Academy Awards of Merit for achievements during 2010\%. Rule sixteen. p. 18-19. http://www.oscars.org/awards/academyawards/rules/ruleo1.html [consulté le 17 mai 2011]

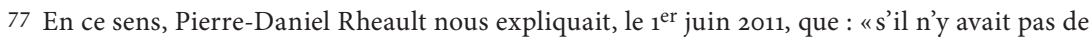
limite de temps à la diffusion télévisée du Gala, on pourrait ajouter une catégorie, pour la musique documentaire, dont le processus de création n’a rien à voir avec la musique des fictions ». Aussi, notons que l'Académie Canadienne du Cinéma et de la Télévision (ACCT) offre depuis 2002 des prix Gémeaux soulignant les productions pour la télévision, l'Internet et les nouveaux médias, trois catégories distinctes en musique à l'image : «Meilleure musique originale : dramatique», «Meilleure musique originale : documentaire» et «Meilleur thème musical : toutes catégories». Les Gémeaux offrent des prix en musique pour la télévision depuis leur création, en 1987.

78 «Films publicitaires» est une catégorie qui comprend notamment les publicités télévisuelles, les films faits pour les entreprises privées (film corporatif) et les bandes-annonces pour le cinéma.

79 Par exemple, en 2010, Gameloft Montréal a plus d'une douzaine de compositeurs salariés, travaillant à temps plein, pour écrire la musique de ses jeux vidéo. De plus, Montréal est passée au $5^{\mathrm{e}}$ rang des villes les plus importantes en jeux vidéo, en 2009, selon le président du conseil d'administration de l'Alliance Numérique, et président du conseil \& chef de la direction chez Druide Numérique, André d'Orsonnens, dans la publication du programme officiel du Sommet International du Jeu vidéo de Montréal de 2009. Aussi, lors de son allocution devant la chambre de commerce de Montréal au Hilton Bonaventure, mardi 15 mars 2011, le patron d'Ubisoft Montréal et Toronto, Yannis Mallat, témoignait d'une croissance annuelle moyenne de $23 \%$ dans l'industrie montréalaise du jeu. Repris sur canoe.ca par Jean-François Cloutier dans l'article: «Ubisoft brandit le spectre de Vancouver», publié le $1^{\text {er }}$ juin 2011. 
ACCT. 2009. 31st Annual Genie Awards. 2010 Rules \& Regulations. http:// www.genieawards.ca [consulté le 17 mai 2011]

ADISQ. 2010a. «Réglementation des Félix. Telle qu’adoptée par le conseil d’administration de l'ADISQ en date du 17 mars 2010 ». Obtenu de http://www. adisq.com

ADISQ. 201ob. Site internet de l'Association québécoise de l'industrie du disque, du spectacle et de la vidéo. http://www.adisq.com [consulté le 12 septembre, 2010]

American Film Institute. 2005. 100 years of films scores http://www.afi. com/10oyears/scores.aspx [consulté le 27 mai, 2011].

AMPAS. Academy of Motion Picture Arts and Sciences Awards Database. http://awardsdatabase.oscars.org [consulté le 12 septembre 2010]

Blais, M.-C. 18 février 2010. Jorane : le rappel infini, Montréal : La Presse.

Céré, Jean-Christian. 17 mai 2011. Conversation téléphonique entre Jean-Christian Céré et Mathieu Lavoie.

Chion, Michel. 1995. La musique au cinéma. Paris : Fayard.

Cloutier, Jean-François. $1^{\text {er }}$ juin 2011. "Ubisoft brandit le spectre de Vancouver», Argent. http://argent.canoe.ca

Cooke, Mervyn. 2008. A history of film music. Cambridge, UK; New York: Cambridge University Press.

Corigliano, John. (s.d.) Site web de John Corigliano. http://www.johncorigliano.com [consulté le 13 septembre 2010].

Corriveau, Michel. (s.d.) Site web de Michel Corriveau. http://michelcorriveau. ca [consulté le 30 mai 2011].

— 30 mai 2011. Correspondance personnelle par courriel entre Michel Corriveau et Mathieu Lavoie.

De Repentigny, Alain. 19 février 2010. Jorane en spectacle: une voix, omniprésente, Montréal : La Presse.

D’Orsonnens, André. 2009. Programme officiel du Sommet International du Jeu vidéo de Montréal de 2009. Montréal : Aliance Numérique.

Gagnon, Annie Joan. (s.d.) «Daniel Bélanger". Encyclopédie de la musique au Canada. http://www.thecanadianencyclopedia.com. [consulté le 31 mai 2011].

Grégoire, Richard. $1^{\text {er }}$ juin 2011. Correspondance personnelle par courriel entre Richard Grégoire et Mathieu Lavoie.

Hickman, Roger. 2006. Reel music : exploring 100 years of film music. New York : W.W. Norton.

Internet Movie Database. (s.d.). http://www.imdb.com

Karlin, Fred. 1994. Listening to movies : the film lover's guide to film music. New York \& Toronto : Schirmer Books.

La Grande Nuit du cinéma. 2008. La Soirée des Jutra. Prix Jutra 2008-Longs Métrages / Fictions. Premier Bulletin de vote pour le choix des finalistes. Catégorie $\mathrm{n}^{\mathrm{O}} 13$ «Meilleure Musique».

La Grande Nuit du cinéma. 2010. Finalistes et gagnants de la $13^{\mathrm{e}}$ soirée des Jutra. http://www.lesjutra.ca/nominations.php [consulté le 14 septembre 2010]. 
Lack, Russel. 1997. Twenty four frames under : a buried history of film music. London: Quartet Books.

Latreille, Marie-France. 2008. «La représentation de la chanson québécoise dans le cinéma québécois ». Mémoire de maîtrise, Université de Montréal.

Le Gramophone Virtuel. (s.d.) Willie Eckstein, pianiste et compositeur (18881963). http://www.collectionscanada.gc.ca/gramophone/o28011-1008-f.html [consulté le $1^{\mathrm{er}}$ juin, 2011].

Lever, Yves et Pierre Pageau. 2006. Chronologie du cinéma au Québec, 18942004. Montréal: Les 400 coups.

Major, Catherine. Site web de Catherine Major. (s.d.). http://catherinemajor .com [consulté le 31 mai 2011].

Prendergast, Roy M. 1977. Film music : a neglected art : a critical study of music in films. New York: W. W. Norton.

Rheault, Pierre-Daniel. $1^{\text {er }}$ juin 2011. Correspondance personnelle par courriel entre Pierre-Daniel Rheault et Mathieu Lavoie.

St-Pierre, Luc. 2 juin 2011. Correspondance personnelle par courriel entre Luc St-Pierre et Mathieu Lavoie.

Tagg, Phillip. 1982. «Analysing Popular Music». Popular Music, ${ }^{\circ} \mathrm{O} 2: 4$.

The Academy of Motion Picture Arts and Sciences. (2010). Rules for the 83rd annual Academy Awards of Merit for achievements during 2010. Los Angeles \& Beverly Hills.

Thérien, Robert. 2008. "Cinéma Cinéma: les plus belles chansons du cinema quebecois». Pochette du CD. Montréal : Musicor.

Trépanier-Jobin, Gabrielle. 2008. «Le cinéma québécois: un succès réel ou imaginé?» Nouvelles "Vues" sur le cinéma Québécois, nº. www.cinemaquebecois.net.

Welsh, Henry. 9 mai 2008. Correspondance personnelle par courriel entre Henry Welsh et Mathieu Lavoie.

\section{RÉSUMÉ}

Aux Jutra, concours récompensant l'industrie du cinéma québécois, on assiste aujourd'hui aux premières années d'une forme de reconnaissance réfléchie de la musique de film et des compositeurs œuvrant dans ce domaine. C'est le résultat de nombreux débats - voire controverses - dans le milieu professionnel, en ce qui concerne la crédibilité du processus d'attribution des prix dans la catégorie "Meilleure musique». En étudiant les résultats du concours depuis sa création en 1999 et en comparaison avec les règlements et l'historique des prix des Félix, des Génies et surtout des Oscars, nous montrerons que les mises à jour récentes des règles d'admissibilité des musiques aux Jutra sont justifiées, mais aussi qu'elles doivent être améliorées. L’un des objectifs de cet article sera de comprendre ce que représente la notion d'originalité en musique de film au regard d'une compétition dans l'industrie du cinéma québécois. Un deuxième objectif sera de questionner l'importance de reconnaître, dans des catégories distinctes, les musiques filmiques instrumentales «dramatiques» de celles davantage de style populaire ainsi que des chansons, lorsque celles-ci sont originales. Aussi, on s'interrogera sur l'impact de la popularité préétablie d'un artiste sur ses chances de remporter 
un prix pour son travail. Finalement, à partir des nouveautés aux Jutra de 2011, nous esquisserons certaines conséquences pour l’avenir de la compétition.

\begin{abstract}
The Jutra Awards, an annual competition rewarding Quebec's film industry, are in their first years of a renewed and improved approach to celebrating film music and its composers. This is the result of much debate-even controversy-in the industry, with respect to the credibility of the awards in the "Best Music" category. By studying the results of the competition since its inception in 1999 and in comparison with the regulations and the histories of the Felix, the Genie, and especially of the Academy Awards, we will show that the recent updates to the rules of eligibility for music at the Jutras are justified, but also that they can still be improved upon. One objective of this paper is to better understand the concept of originality in film music, as it pertains to competition in the film industry in Quebec. A second objective will be to question the importance of recognizing, in separate categories, "dramatic" orchestral film scores, original popular music and original songs. Also, we will consider the influence of an artist's pre-established popularity on his chances of winning an award for his work. Finally, based on the rule changes made for the 2011 Jutras, we will outline some implications for the future of the competition.
\end{abstract}

